\title{
Article
}

\section{The Magnetic Properties of Fe/Cu Multilayered Nanowires: The Role of the Number of Fe Layers and Their Thickness}

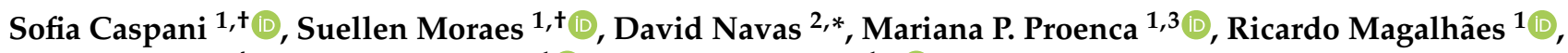 \\ Cláudia Nunes ${ }^{4}$, João Pedro Araújo ${ }^{1}\left[\right.$ and Célia T. Sousa ${ }^{1, *(1)}$ \\ 1 IFIMUP and Departamento de Física e Astronomia, Faculdade de Ciências Universidade do \\ Porto, Rua do Campo Alegre 687, 4169-007 Porto, Portugal; sofixsofi@gmail.com (S.C.); \\ suellen.fisica@gmail.com (S.M.); mpproenca@fc.up.pt (M.P.P.); ricardo.magalhaes@fc.up.pt (R.M.); \\ jearaujo@fc.up.pt (J.P.A.) \\ 2 ICMM-CSIC-Instituto de Ciencia de Materiales de Madrid, Sor Juana Inés de la Cruz 3, 28049 Madrid, Spain \\ 3 ISOM and Dpto. Electrónica Física, Universidad Politécnica de Madrid, Avda. Complutense 30, \\ 28040 Madrid, Spain \\ 4 LAQV, REQUIMTE, Faculty of Pharmacy of Porto University, 4050-313 Porto, Portugal; cdnunes@ff.up.pt \\ * Correspondence: davidnavasotero@gmail.com (D.N.); celiasousa@fc.up.pt (C.T.S.); \\ Tel.: +34-913-349-000 (D.N.); +35-122-40-2337 (C.T.S.) \\ + These authors have contributed equally to this work and both should be considered as first authors.
}

check for updates

Citation: Caspani, S.; Moraes, S.; Navas, D.; Proenca, M.P.;

Magalhães, R.; Nunes, C.; Araújo, J.P.; Sousa, C.T. The Magnetic Properties of Fe/Cu Multilayered Nanowires: The Role of the Number of Fe Layers and Their Thickness. Nanomaterials 2021, 11, 2729. https://doi.org/ $10.3390 /$ nano11102729

Academic Editor: Albert G. Nasibulin

Received: 15 September 2021

Accepted: 12 October 2021

Published: 15 October 2021

Publisher's Note: MDPI stays neutral with regard to jurisdictional claims in published maps and institutional affiliations.

Copyright: (c) 2021 by the authors. Licensee MDPI, Basel, Switzerland. This article is an open access article distributed under the terms and conditions of the Creative Commons Attribution (CC BY) license (https:/ / creativecommons.org/licenses/by/ $4.0 /)$.

\begin{abstract}
Multi-segmented bilayered Fe/Cu nanowires have been fabricated through the electrodeposition in porous anodic alumina membranes. We have assessed, with the support of micromagnetic simulations, the dependence of fabricated nanostructures' magnetic properties either on the number of $\mathrm{Fe} / \mathrm{Cu}$ bilayers or on the length of the magnetic layers, by fixing both the nonmagnetic segment length and the wire diameter. The magnetic reversal, in the segmented Fe nanowires (NWs) with a $300 \mathrm{~nm}$ length, occurs through the nucleation and propagation of a vortex domain wall (V-DW) from the extremities of each segment. By increasing the number of bilayers, the coercive field progressively increases due to the small magnetostatic coupling between Fe segments, but the coercivity found in an Fe continuous nanowire is not reached, since the interactions between layers is limited by the $\mathrm{Cu}$ separation. On the other hand, Fe segments $30 \mathrm{~nm}$ in length have exhibited a vortex configuration, with around $60 \%$ of the magnetization pointing parallel to the wires' long axis, which is equivalent to an isolated Fe nanodisc. By increasing the Fe segment length, a magnetic reversal occurred through the nucleation and propagation of a V-DW from the extremities of each segment, similar to what happens in a long cylindrical Fe nanowire. The particular case of the $\mathrm{Fe} / \mathrm{Cu}$ bilayered nanowires with Fe segments $20 \mathrm{~nm}$ in length revealed a magnetization oriented in opposite directions, forming a synthetic antiferromagnetic system with coercivity and remanence values close to zero.
\end{abstract}

Keywords: nanowires; porous anodic alumina membranes; Fe/Cu bilayers; magnetization reversal

\section{Introduction}

Considerable interest has arisen recently in studying 1D nanostructures, such as nanowires, nanopillars, and nanorods, owing to their potential applications [1,2]. The term nanowires (NWs) describes wires with a large length-to-diameter ratio, i.e., aspect ratio. Some of their remarkable properties arise from having a high density of electronic states, diameter-dependent band gaps, an enhanced surface scattering of electrons and photons, and high surface-to-volume ratios [3-5]. These properties lead to a unique electrical, optical, and magnetic behavior, making them suitable for many industrial and medical applications $[2,6]$. In addition, cylindrical NWs have been suggested as key elements for the development and understanding of a new research field known as magnetism in curved geometries [7]. It was recently demonstrated that the curved geometry of NWs can lead to novel and non-trivial magnetic phenomena, such as the formation of skyrmion magnetic configurations [8,9] and Bloch-point domain walls [10,11]. 
Among the several methods that can be employed to synthesize cylindrical NWs [12], template-assisted electrodeposition in porous anodic aluminum oxide (AAO) is one of the most reported techniques. The main advantages are its simplicity of controlling the size and shape of the nanostructures, without requiring expensive equipment or timeconsuming processes $[13,14]$. Moreover, AAO templates allow for the fabrication of large areas of highly ordered nanostructures, which is particularly important when studying order-dependent properties such as magnetic responses.

The magnetic behavior of ferromagnetic NW arrays grown in AAO templates has been extensively investigated over the last two decades $[15,16]$. Besides single material-based NWs, multi-segmented NWs have gained increasing attention for applications in magnetic devices $[17,18]$. In particular, multilayered nanostructures, composed of several magnetic and non-magnetic materials, offer the possibility of restraining the magnetic interactions and tuning the magnetic anisotropic effects by changing the deposited material and/or the segments' length $[17,19]$.

The effect of the length of the magnetic layer on the magnetic behavior of cylindrical nanostructures has been largely investigated in Ni/Cu [17,20-24], Ni/Au [25], $\mathrm{Co} / \mathrm{Cu}$ [26-28], $\mathrm{CoFe} / \mathrm{Au}$ [29], $\mathrm{CoFe} / \mathrm{Cu}$ [30] $\mathrm{CoNi} / \mathrm{Cu}$ [31], and $\mathrm{CoFeB} / \mathrm{Cu}$ [32] multilayered NWs. On the other hand, complementary studies have reported the magnetic behavior of multi-segmented NWs with tuned lengths of the non-magnetic layer, e.g., $\mathrm{Co} / \mathrm{Cu}[27,33]$, $\mathrm{Ni} / \mathrm{Cu}[21,23,34], \mathrm{CoFe} / \mathrm{Au}$ [29], $\mathrm{CoFe} / \mathrm{Cu}$ [35] $\mathrm{FeCoCu} / \mathrm{Cu}$ [36], $\mathrm{FeCo} / \mathrm{Cu}$ [37], and $\mathrm{CoFeB} / \mathrm{Cu}[32]$ nanostructures. These works have demonstrated that the magnetic behavior of the multilayered nanoarchitectures can be selectively tuned according to the required applications [20,38-40]. However, to employ such structures in biomedical applications, and since Co and Ni possess high toxicity levels, the need to fabricate completely biocompatible segmented NWs has arisen.

In this framework, few studies have been reported. Almasi-Kashi et al. [41] studied the magnetic properties of multi-segmented $\mathrm{Fe} / \mathrm{Cu}$ NWs with very thin $\mathrm{Cu}$ spacer layers $(<10 \mathrm{~nm})$, corresponding to interacting magnetic layers. In a complementary experiment, Moraes et al. [19] investigated the role of the $\mathrm{Cu}$ segment's length in multilayered $\mathrm{Fe} / \mathrm{Cu}$ NWs. Their results showed that, for short $\mathrm{Cu}$ segments $(<30 \mathrm{~nm})$, the magnetic properties of the arrays were mainly controlled by the magnetostatic coupling between magnetic segments, behaving like a homogeneous array of Fe NWs. On the contrary, these nanoarchitectures behaved as an ensemble of non-interacting elements along the NW axis for longer non-magnetic spacers.

Therefore, in this work, we report the synthesis by template-assisted electrodeposition in porous $\mathrm{AAO}$ and the characterization of $\mathrm{Fe} / \mathrm{Cu}$ multi-segmented NWs. We have investigated the changes in the magnetic behavior of multi-segmented $\mathrm{Fe} / \mathrm{Cu}$ NWs resulting from varying the number of bilayers from 1 to 20, for fixed $\mathrm{Fe}$ and $\mathrm{Cu}$ lengths, or changing the length of the magnetic layer for two $\mathrm{Cu}$ layer lengths $(60$ and $120 \mathrm{~nm})$. In addition, the Fe lengths have been substantially varied (from 20 to $345 \mathrm{~nm}$ ) to allow for a better understanding of the magnetic properties of the nanostructures when modifying their aspect ratio.

\section{Materials and Methods}

$\mathrm{Fe} / \mathrm{Cu}$ multi-segmented NWs were fabricated by electrochemical deposition from a single aqueous bath, using AAO templates as the working electrode. The AAO membranes were prepared from high-purity $(>99.999 \%) \mathrm{Al}$ foils by a standard two-step anodization process [16]. The first and second anodization steps lasted 24 and $48 \mathrm{~h}$, respectively, having been performed at a constant voltage of $40 \mathrm{~V}$ in a $0.3 \mathrm{M}$ oxalic acid solution, which was kept at a temperature of $\approx 2{ }^{\circ} \mathrm{C}$. Through this process, self-organized AAO templates with a $1 \mathrm{~cm}$ diameter, a length of $120 \mu \mathrm{m}$, and pore diameter and interpore distances of $d \approx 35 \pm 5 \mathrm{~nm}$ and $D_{\text {int }} \approx 105 \pm 5 \mathrm{~nm}$, respectively, were obtained.

The $\mathrm{Fe} / \mathrm{Cu}$ NWs were grown at room temperature by a DC pulsed electrodeposition method, as described in [19]. The used electrolyte contained $0.4 \mathrm{M} \mathrm{H}_{3} \mathrm{BO}_{3}, 0.19 \mathrm{M}$ 
$\mathrm{FeSO}_{4} \cdot 7 \mathrm{H}_{2} \mathrm{O}, 0.005 \mathrm{M} \mathrm{CuSO}_{4} \cdot 5 \mathrm{H}_{2} \mathrm{O}$, and $0.003 \mathrm{M}$ ascorbic acid $\left(\mathrm{C}_{6} \mathrm{H}_{8} \mathrm{O}_{6}\right)$, and the electrodeposition was carried out by applying a $-1.1 \mathrm{~V}$ versus $\mathrm{Ag} / \mathrm{AgCl}$ reference electrode pulse to grow the Fe layer, followed by a pulse of $-0.6 \mathrm{~V}$ to grow the $\mathrm{Cu}$ spacer.

To assess the morphological properties of the fabricated nanostructures, scanning electron microscopy (SEM) analysis has been performed by using an FEI Inspect F50 microscope (FEI Europe, Madrid, Spain). To investigate the structural properties of the wires, X-ray diffraction measurements in Bragg Brentano geometry have been executed through the use of a Rigaku SmartLab diffractometer (Rigaku Corporation, Tokio, Japan) with $\mathrm{Cu}-\mathrm{K} \alpha$ radiation $(1.540593 \AA), 45 \mathrm{kV}$, and $200 \mathrm{~mA}$. The magnetic hysteresis loops of Fe/ $\mathrm{Cu}$ NW arrays have been measured with a vibrating sample magnetometer (VSM, LakeShore Controller, Model 7304) (LakeShore, Westerville, OH, USA). All measurements have been performed at room temperature, with the magnetic field applied parallel and perpendicular to the NWs' long axis.

To better understand the magnetic results, 3-D micromagnetic simulations were also performed using the MuMax3 software (Version 3.9.1, DyNaMat group, Ghent University, Belgium) [42]. Due to the small amount of $\mathrm{Cu}$ contamination in the electrodeposited Fe segments, the magnetization of the Fe layers was set to $M_{\text {Sat }}=1600 \mathrm{emu} / \mathrm{cm}^{3}$, instead of the typical saturation magnetization value for $F e\left(1700 \mathrm{emu} / \mathrm{cm}^{3}\right)$ [43]. While we used 0.5 as the damping parameter to ensure the rapid convergence of the simulations, the Fe exchange coupling constant and magnetocrystalline anisotropy values were fixed to $A=43 \times 10^{-8} \mathrm{erg} / \mathrm{cm}[44,45]$ and $K=4.8 \times 10^{5} \mathrm{erg} / \mathrm{cm}^{3}$, respectively. As the exchange length of our material is $l_{e x}=\sqrt{2 A / \mu_{0} M_{\text {Sat }}^{2}} \approx 5 \mathrm{~nm}$, the cell size was chosen to be $(2.5 \times 2.5 \times 2.5) \mathrm{nm}^{3}$.

\section{Results and Discussion}

\subsection{Electrochemical Growth}

A representative example of the potential sequence employed for the electrodeposition of multi-segmented $\mathrm{Fe} / \mathrm{Cu}$ NWs is shown in Figure 1a,b. The possibility to perform a codeposition of $\mathrm{Fe} / \mathrm{Cu}$ layers from a single bath is based on the difference between the reduction potentials of $\mathrm{Cu}(+0.14 \mathrm{~V}$ vs. $\mathrm{Ag} / \mathrm{AgCl}$ electrode) and $\mathrm{Fe}(-0.644 \mathrm{~V}$ vs. $\mathrm{Ag} / \mathrm{AgCl}$ electrode) and on the very low concentration of $\mathrm{Cu}$ ions in the deposition solution $\left(\left[\mathrm{Cu}^{2+}\right]\right.$ $\left.<<\left[\mathrm{Fe}^{2+}\right]\right)$. These conditions ensure multilayers of $\mathrm{Fe} / \mathrm{Cu}$ with low contamination since, for low $\mathrm{Cu}^{2+}$ concentrations, the $\mathrm{Cu}$ deposition is diffusion-limited over a wide potential range (from -0.15 to $-0.7 \mathrm{~V}$ ) $[24,46,47]$. Therefore, for an Fe deposition at high potentials $(-1.1 \mathrm{~V})$, the molar fraction of $\mathrm{Cu}$ in Fe layers is expected to be lower than $10 \%$. Figure 1a shows the pulses required to grow $15 \mathrm{Cu} / \mathrm{Fe}$ bilayers and confirms the homogeneous growth of both layers.

The nucleation and growth mechanisms of the nanostructures, as well as their electrodeposition rates, can be determined using the current transients (Figure 1c,d). In general, the nucleation of each metal layer mainly depends on the nature of the previously electrodeposited layer and on the density of active sites on its surface, such as steps, kinks, or other defects, whereas its growth mechanism depends on the applied potential and electrolyte [47]. Depending on the nucleation rate, two limiting cases can be identified, i.e., instantaneous nucleation and progressive nucleation, which correspond to high and low nucleation rates, respectively [48,49]. In both cases, the current density starts to increase with time due to the 3D growth of the nucleation centers, which leads to an increase in the electrodeposition surface area. The current then achieves a quasi-constant value when the diffusion zones around the growing nuclei start to overlap, resulting in a 1D diffusion-limited current [48]. 

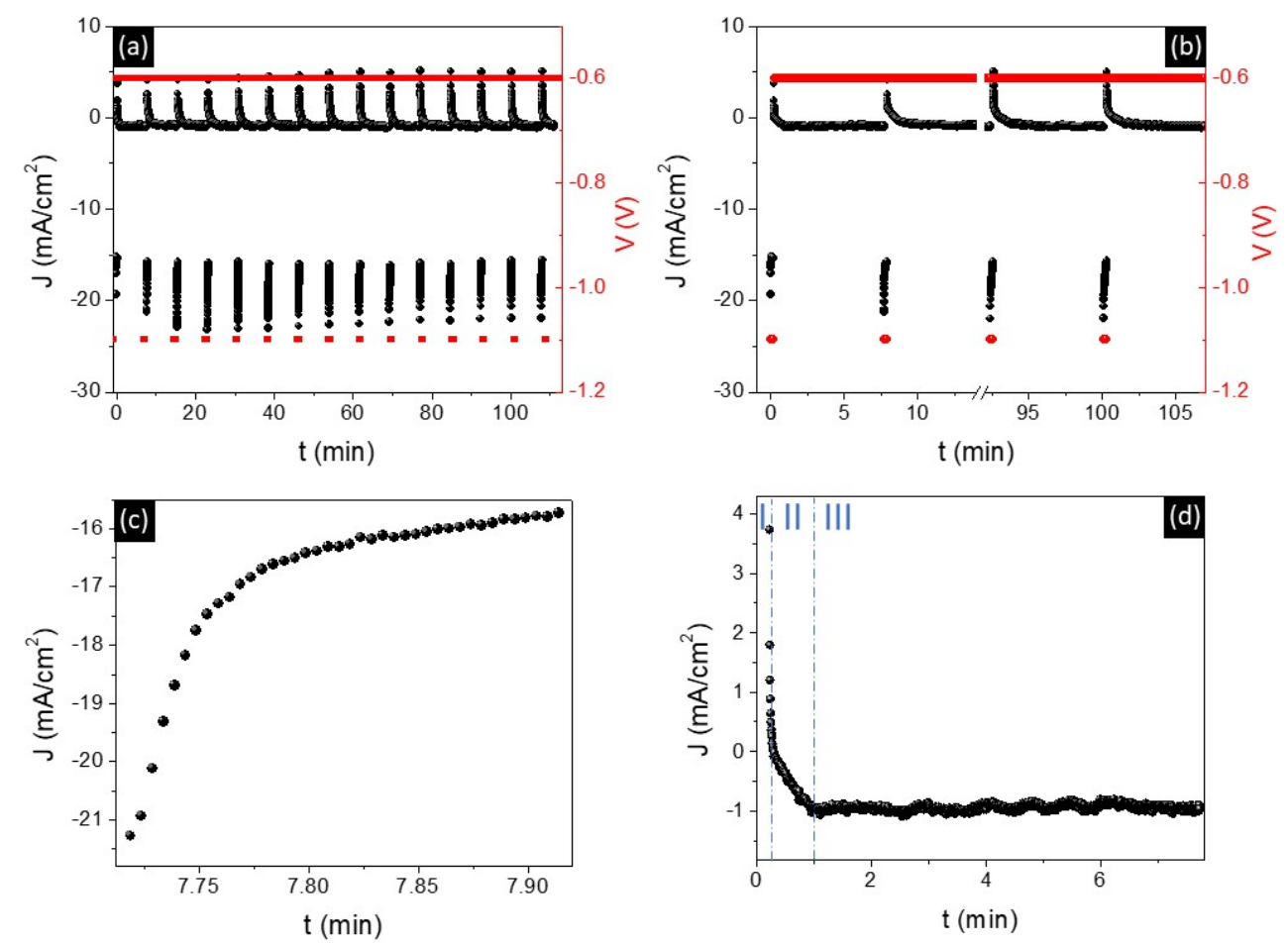

Figure 1. Current density transients of the pulsed electrodeposition process for the synthesis of the multi-segmented $\mathrm{Fe} / \mathrm{Cu}$ nanowires (NWs) in anodic aluminum oxide (AAO) templates: (a) $15 \mathrm{Fe}$ deposition pulses at $-1.1 \mathrm{~V}$ and $15 \mathrm{Cu}$ deposition pulses at $-0.6 \mathrm{~V}$. (b) Zoom of the first two and last two pulses of the Fe and $\mathrm{Cu}$ electrodeposition. Selected current density transients during the (c) Fe deposition and (d) $\mathrm{Cu}$ deposition processes.

In the deposition of Fe layers (Figure 1c), a decrease in the absolute value of the current density is observed, which is associated with diffusion-limited growth. However, the expected initial increase in currents due to nucleation is not observed, due to the high deposition rate of $\mathrm{Fe}$ associated with the high deposition potential applied $(-1.1 \mathrm{~V})$. This fact indicates the occurrence of an instantaneous nucleation, where the nucleation sites become saturated after short times [50]. Regarding $\mathrm{Cu}$, a more complex $J(t)$ curve is observed (Figure 1d). In this case, the positive current observed in the first several seconds can be related to the oxidation of the previous Fe layer (Phase I). The absolute value of the current density transients then increases due to the $\mathrm{Cu}$ layer nucleation (Phase II), followed by a quasi-constant current density during the $\mathrm{Cu}$ layer growth (Phase III). Taking into account this type of curve, together with the quite low deposition potential of $\mathrm{Cu}$ $(-0.6 \mathrm{~V})$ and the reduced concentration of $\mathrm{Cu}$ ions in the electrolyte, the Cu nucleation can be associated with a progressive nucleation behavior, where the density of nuclei increases linearly with time due to the low deposition rate $[49,50]$.

Moreover, the total charge transients, $Q(t)$, led us to determine deposition rates of $(0.322 \pm 0.001)$ and $(0.0037 \pm 0.0003) \mathrm{nm} / \mathrm{min}$ for the Fe and Cu layers, respectively. The low deposition rate of $\mathrm{Cu}$ compared with $\mathrm{Fe}$ is mainly associated with the low concentration of $\mathrm{Cu}$ ions present in the solution. To explore the magnetic behavior of the segmented NWs as a function of the number of layers and the length of the Fe layers, different numbers of pulses and deposition times have been tested.

\subsection{Morphological and Structural Characterization}

Representative SEM cross-sectional images of the multilayered Fe/Cu NW arrays inside the AAO template are shown in Figure 2. The brighter and darker segments along the wires correspond to the $\mathrm{Cu}$ and Fe segments, respectively. Figure 2 illustrates representative 
NWs samples with highly homogeneous and well-defined segments, possessing $\mathrm{Cu}$ layer lengths of 60 and $120 \mathrm{~nm}$, and Fe lengths varying between 20 and $60 \mathrm{~nm}$.
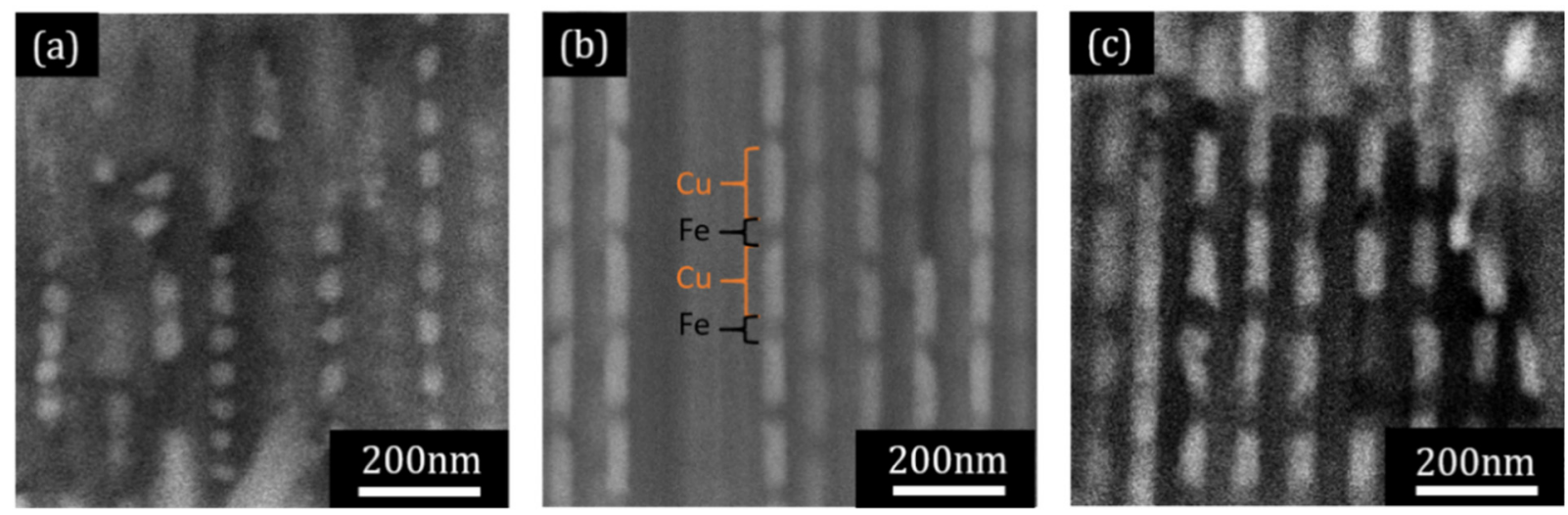

Figure 2. SEM cross-sectional views of multilayered $\mathrm{Fe} / \mathrm{Cu}$ NWs embedded in AAO templates, exhibiting average $\mathrm{Cu}$ lengths of (a) $60 \pm 7 \mathrm{~nm}$ and (b,c) $120 \pm 5 \mathrm{~nm}$ and Fe lengths of (a) $20 \pm 5 \mathrm{~nm},(\mathbf{b}) 30 \pm 3 \mathrm{~nm}$, and (c) $60 \pm 8 \mathrm{~nm}$.

X-ray diffraction (XRD) measurements (data not shown) of Fe NWs revealed a polycrystalline body-centered cubic (bcc) structure, with an intense peak corresponding to a preferential direction along the (110) crystallographic plane. The $\mathrm{Cu}$ layers presented a polycrystalline fcc structure, exhibiting a main peak corresponding to the (111) crystallographic plane.

\subsection{Magnetic Properties}

The resulting magnetic hysteresis loops of $\mathrm{Fe} / \mathrm{Cu} \mathrm{NW}$ arrays are presented and analyzed in the following sections, having been measured both in the parallel and perpendicular direction with respect to the wires' long axis.

\subsubsection{Varing the Number of Bilayers}

In this section, we assess the magnetic behavior of segmented NWs as a function of the bilayers' number $(N)$. The prepared samples consisted of $N=1,3,5,15$, and 20 bilayers, with Fe lengths of $\sim 300 \mathrm{~nm}$ and $\mathrm{Cu}$ segments with a length of $\sim 120 \mathrm{~nm}$. Previous works have shown that, when increasing the length of the non-magnetic layer above the wire diameter value, the interactions between the magnetic layers along the wire become negligible and the system behaves as an ensemble of non-interacting entities [19,51]. The magnetic hysteresis loops for an Fe NW array ( $3 \mu \mathrm{m}$ in length) and the Fe/Cu NWs with 1 , 5 , and 20 bilayers are presented in Figure 3. In all cases, an increase in the coercivity and remanence values is observed when the magnetic field is applied parallel to the wire's axis, confirming the anisotropic behavior of the structures. A similar magnetic behavior has been observed for all samples, being independent of the number of bilayers.

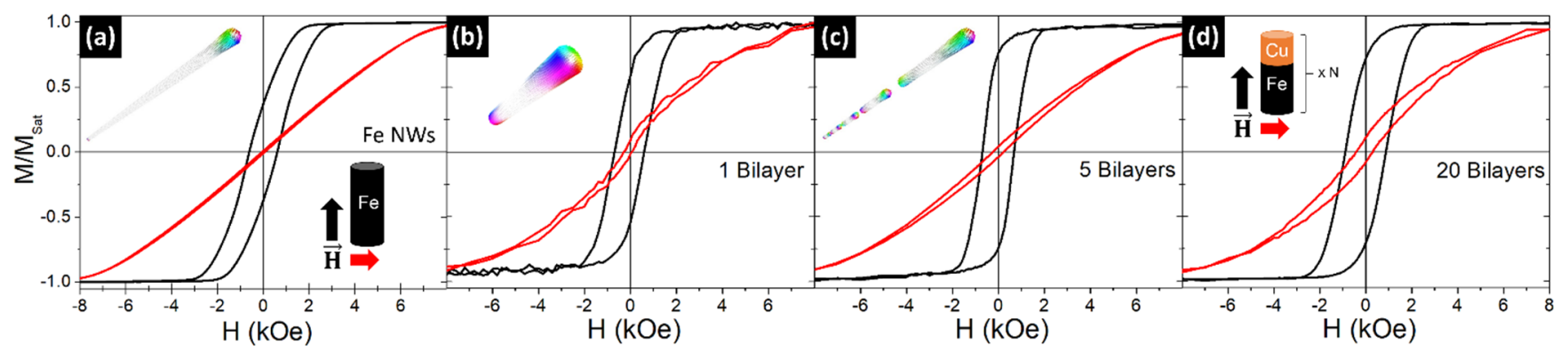

Figure 3. Magnetic hysteresis loops of (a) Fe NW arrays with $45 \mathrm{~nm}$ in diameter and $3000 \mathrm{~nm}$ in length and (b-d) Fe/Cu NW arrays with $45 \mathrm{~nm}$ in diameter, Fe lengths of $\sim 300 \mathrm{~nm}$, Cu segment lengths of $\sim 120 \mathrm{~nm}$, and (b) 1, (c) 5, and (d) 20 bilayers, measured along the parallel (black) and perpendicular (red) directions. Insets show the respective 3D simulated magnetic configurations at the switching field state, when applying the magnetic field parallel to the wire axis. 
To analyze the magnetization reversal modes in $\mathrm{Fe} / \mathrm{Cu} \mathrm{NWs}$ as a function of the number of bilayers, 3-D micromagnetic simulations using the MuMax3 software (Version 3.9.1) [42] were performed. Following the experimental results and our previous work [19], multisegmented individual NWs $40 \mathrm{~nm}$ in diameter, with ferromagnetic Fe layers $300 \mathrm{~nm}$ in length and non-magnetic $\mathrm{Cu}$ spacers $120 \mathrm{~nm}$ in length, were simulated. The number of bilayers was varied from 1 to 15 . An individual long Fe NW ( $3 \mu \mathrm{m}$ in length) was also simulated for comparison (inset in Figure 3a).

In agreement with the literature [52], micromagnetic simulations show that the magnetization reversal process in long cylindrical Fe NWs ( $3 \mu \mathrm{m}$ in length) occurs through the nucleation and propagation of a vortex domain wall (V-DW) from the NW extremities (see inset in Figure 3a). Similarly, the magnetic reversal in the $300 \mathrm{~nm}$ length segmented Fe NWs occurs through the nucleation and propagation of a V-DW from the extremities of each segment, regardless of the number of bilayers (see insets in Figure $3 b, c$ ).

Figure $4 \mathrm{a}, \mathrm{b}$ presents the normalized remanence and coercivity values, respectively, as a function of the number of $\mathrm{Fe} / \mathrm{Cu}$ bilayers. The coercivity values were found to slightly increase with the number of bilayers, either when the field is applied parallel or perpendicular to the wire's axis. On the other hand, the remanence values are almost constant along the perpendicular direction, while they seem to first increase with the number of bilayers (up to 5 bilayers) and then keep constant for longer NWs (5-20 bilayers). This phenomenon can be associated with the complex behavior of the magnetization reversal mechanism in multilayered structures, in which interactions between neighboring NWs must be considered $[17,19]$.
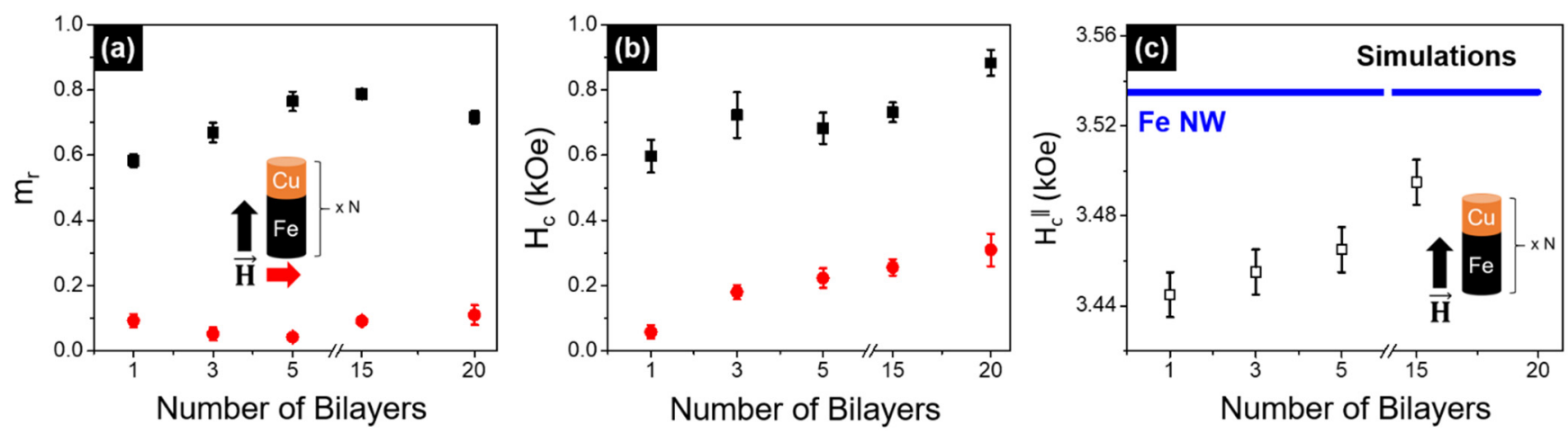

Figure 4. (a) Normalized remanence $\left(m_{r}\right)$ and $(\mathbf{b})$ coercivity $\left(H_{c}\right)$ values as a function of the number of Fe/Cu bilayers, measured when applying a magnetic field parallel (black squares) and perpendicular (red dots) to the wire's axis. (c) Coercive field of the segmented Fe/Cu NWs, as a function of the number of layers (open symbols), and of the $3 \mu \mathrm{m}$ length isolated Fe NW (blue continuous line) extracted from the simulated hysteresis loops when the external field was applied parallel to the NW axis.

From the simulated hysteresis loops of the isolated Fe/Cu NWs with the magnetic field applied along the wire axis, we have extracted the coercivity fields $\left(H_{C}\right)$ as a function of the number of bilayers (open symbols in Figure 4c) and for the long Fe NWs (continuous blue line in Figure 4c). Despite the significant difference between the $H_{C}$ values obtained experimentally and the simulated ones, a good correlation with the experimental data was achieved (Figure $4 b, c)$. Low-aspect-ratio segmented NWs with 35-nm-length Fe segments present a behavior almost like a set of non-interacting nanoparticles when they are separated by 120-nm-length non-magnetic $\mathrm{Cu}$ spacers [19]. However, when we increase the Fe length to $300 \mathrm{~nm}$, the contribution of the magnetostatic interactions is not negligible, as shown in Figures 3 and 4 . The coercive field progressively increases with the number of bilayers due to the small magnetostatic coupling between Fe segments, but the maximum value obtained does not reach the $H_{C}$ of the continuous $\mathrm{Fe} N W$, since the interaction between layers is limited by the $\mathrm{Cu}$ separation. 


\subsubsection{Varying the Fe Length}

After analyzing the magnetic behavior of $\mathrm{Fe} / \mathrm{Cu} \mathrm{NWs}$ as a function of the number of bilayers, in this section we focus our attention on understanding the influence of the length of the Fe layer on the interactions between the magnetic layers. For that purpose, we have prepared multiple $\mathrm{Fe} / \mathrm{Cu}$ NWs' samples, where the number of bilayers was fixed to 15, and the Fe segments' length was varied between 20 and $300 \mathrm{~nm}$. In addition, the effect of the non-magnetic spacer length has been explored using $\mathrm{Cu}$ segments with lengths of 60 and $120 \mathrm{~nm}$.

Figure 5 presents the magnetic hysteresis loops of the Fe/Cu NW samples with 15 bilayers. As previously reported [53], the magnetic anisotropy in large-aspect-ratio NWs is dominated by the shape anisotropy contribution, leading to the alignment of the easy magnetization axis along the $\mathrm{NWs}^{\prime}$ longitudinal axis (Figure $5 \mathrm{~b}, \mathrm{c}, \mathrm{e}, \mathrm{f}$ ). Regarding the coercivity and remanence values along the parallel direction, a significant increase of both parameters is observed for longer Fe segments, which is accompanied by an increase in the saturation field along the perpendicular direction.

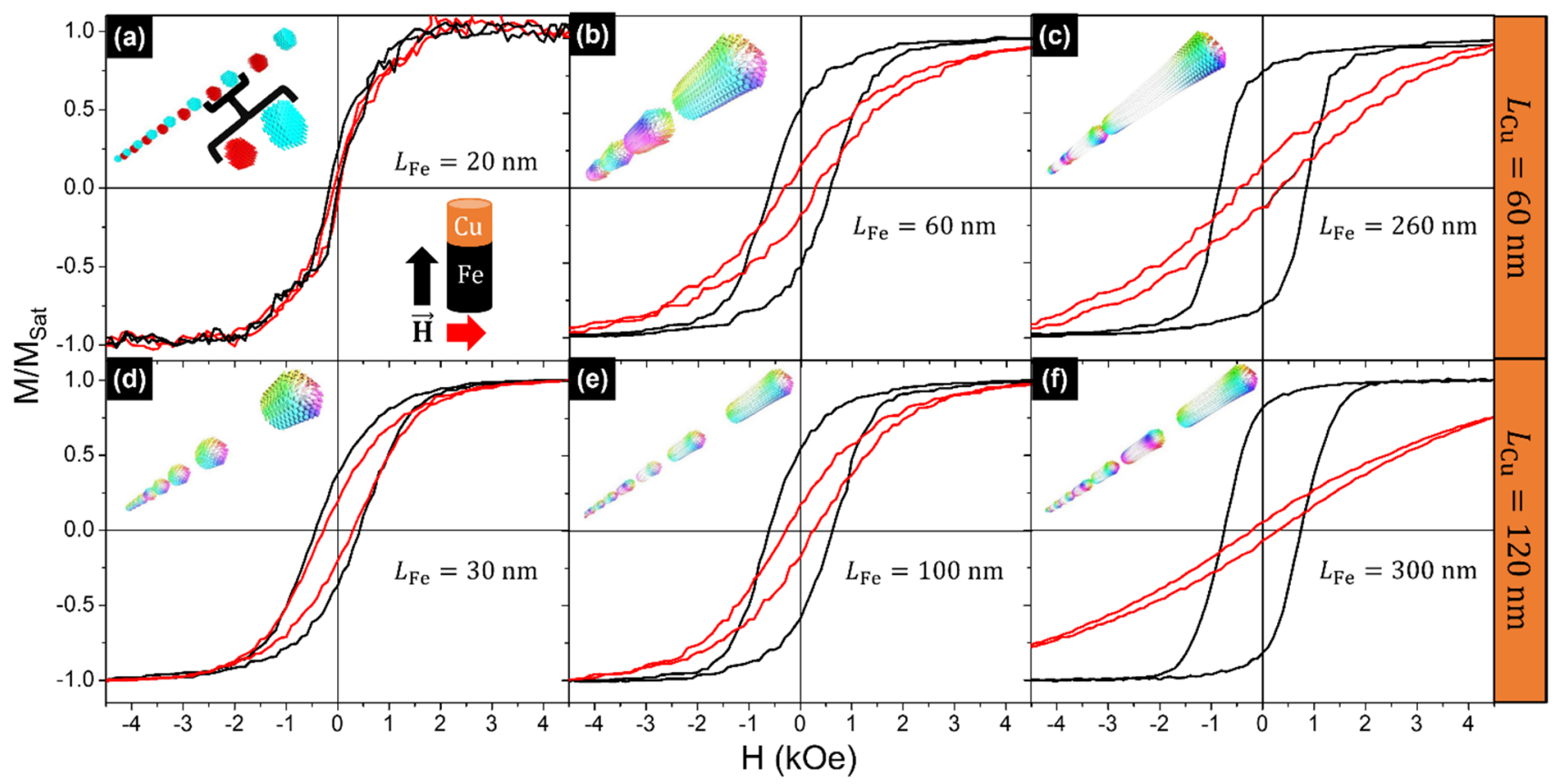

Figure 5. Magnetic hysteresis loops of multi-segmented Fe/Cu NW arrays with different Fe segment lengths $\left(L_{\mathrm{Fe}}\right)$ and $\mathrm{Cu}$ segment lengths $\left(L_{\mathrm{Cu}}\right)$ of $(\mathbf{a}-\mathbf{c}) 60 \mathrm{~nm}$ and $(\mathbf{d}-\mathbf{f}) 120 \mathrm{~nm}$, measured along the parallel (black) and perpendicular (red) directions. Insets show the respective 3D simulated magnetic configurations at the switching field state, when applying the magnetic field parallel to the wire axis.

On the other hand, the hysteresis loops of the NWs with the smallest Fe segments $(20-30 \mathrm{~nm})$, which correspond to aspect ratios $<1$, show that the easy magnetization axis deviates from the longitudinal direction and suggests an isotropic-like behavior (see Figure $5 \mathrm{a}, \mathrm{d})$, in agreement with previous studies $[19,54]$. In addition, a significant difference in the hysteresis loops can be observed when the $\mathrm{Cu}$ segment length is increased from 60 to $120 \mathrm{~nm}$. The easy magnetization axis of Fe/Cu NWs with 20-nm-length Fe segments and 60-nm-length $\mathrm{Cu}$ spacers tends to be perpendicular to the longitudinal wire axis, and the system behaves like a synthetic antiferromagnet, presenting negligible coercivity and remanence values (Figure 5a). On the contrary, and as will be further confirmed by the micromagnetic simulations described below, the easy magnetization axis deviates from the perpendicular direction towards a more parallel one for $\mathrm{Fe} / \mathrm{Cu}$ NWs with $\mathrm{Fe}$ and $\mathrm{Cu}$ segment lengths of $30 \mathrm{~nm}$ and $120 \mathrm{~nm}$, respectively. 
To confirm that the NWs exhibit different magnetization reversal regimes as a function of the Fe segment aspect ratio, the study was complemented by performing 3-D micromagnetic simulations (MuMax3 software, Version 3.9.1) [42]. In this case, we have simulated multi-segmented individual NWs $40 \mathrm{~nm}$ in diameter, varying the Fe layer length from 20 to $300 \mathrm{~nm}$, considering two different lengths for the non-magnetic $\mathrm{Cu}$ spacers (60 and $120 \mathrm{~nm})$ and keeping the total number of bilayers fixed at 15.

The micromagnetic simulations showed that the segmented Fe/Cu NWs behaved like a set of 15 non-interacting nanoparticles when the Fe and $\mathrm{Cu}$ spacer lengths were 30 and $120 \mathrm{~nm}$, respectively (see inset in Figure 5d). In addition, it was confirmed that the 30-nm-length Fe segments (separated by $120 \mathrm{~nm}$ of $\mathrm{Cu}$ ) exhibited a vortex configuration with around $60 \%$ of the magnetization pointing parallel to the NW long axis. As soon as the Fe segment lengths were increased $(\geq 100 \mathrm{~nm}$ ), while keeping the $\mathrm{Cu}$ segments to $120 \mathrm{~nm}$, the magnetic reversal mode occurred through the nucleation and propagation of a V-DW from the extremities of each segment (see insets in Figure 5e,f), similar to what happened in the longer cylindrical Fe NW (inset in Figure 3a). This behavior becomes more evident as the Fe segments' length is increased.

To study the effect of the non-magnetic $\mathrm{Cu}$ spacer layer, $\mathrm{Fe} / \mathrm{Cu}$ NWs with $\mathrm{Cu}$ spacers $60 \mathrm{~nm}$ in length and Fe layers with lengths ranging from 20 to $260 \mathrm{~nm}$ were also simulated. The 3D simulated magnetic configuration at remanence of the Fe/Cu NWs with Fe segments $20 \mathrm{~nm}$ in length showed an easy magnetization axis lying perpendicular to the longitudinal NW's axis (inset in Figure 5a). In addition, the magnetization in consecutive Fe segments is oriented in opposite directions, confirming the formation of a synthetic antiferromagnetic system with coercivity and remanence values close to zero (Figure 5a). As was observed in the samples with $\mathrm{Cu}$ spacer lengths of $120 \mathrm{~nm}$, the magnetization reversal evolved from an in-plane (perpendicular) configuration to the nucleation and propagation of a V-DW from the extremities of each segment for NWs with longer Fe segments ( $\geq 60 \mathrm{~nm})$.

Table 1 summarizes the results obtained, including the lengths of the Fe segments together with the coercivity and normalized remanence values measured along both the parallel and perpendicular directions of the applied field. In addition, the coercivity and reduced remanence values are also presented in Figure 6, as a function of the Fe segments length, considering the external magnetic field applied parallel to the NWs' long axis. Both the coercivity and remanence values were found to progressively increase with increasing Fe length in the multi-segmented Fe/Cu NWs. However, while the parallel coercivity increased until the value corresponding to the long Fe NW was reached (Figure 6b), the remanence values reached even higher values when compared to the continuous Fe NW (Figure 6a). This may be ascribed to the stronger magnetostatic interactions between neighboring wires for the long Fe NWs when compared to multi-segmented Fe/Cu NWs, which decrease the respective remanence values [55].

Table 1. Magnetic properties of multi-segmented NWs: Coercive field $\left(H_{\mathcal{C}}\right)$ and normalized remanence $\left(m_{r}\right)$ measured with the magnetic field applied parallel $(\|)$ and perpendicular $(\perp)$ to the $\mathrm{NWs}^{\prime}$ longitudinal axis.

\begin{tabular}{cccccc}
\hline Systems & $\boldsymbol{L}_{\boldsymbol{F e}}(\mathbf{n m})$ & $\boldsymbol{H}_{\boldsymbol{c}}^{\|}(\mathbf{O e})$ & $\boldsymbol{H}_{\boldsymbol{c}}^{\perp}(\mathbf{O e})$ & $\boldsymbol{m}_{\boldsymbol{r}}^{\|}$ & $\boldsymbol{m}_{\boldsymbol{r}}^{\perp}$ \\
\hline$\left(\mathrm{Fe}_{(20 \mathrm{~nm})} / \mathrm{Cu}_{(60 \mathrm{~nm})}\right)_{15}$ & $20 \pm 5$ & $84 \pm 40$ & $60 \pm 40$ & $0.15 \pm 0.04$ & $0.09 \pm 0.01$ \\
$\left(\mathrm{Fe}_{(60 \mathrm{~nm})} / \mathrm{Cu}_{(60 \mathrm{~nm})}\right)_{15}$ & $60 \pm 7$ & $490 \pm 60$ & $350 \pm 30$ & $0.40 \pm 0.10$ & $0.25 \pm 0.05$ \\
$\left(\mathrm{Fe}_{(260 \mathrm{~nm})} / \mathrm{Cu}_{(60 \mathrm{~nm})}\right)_{15}$ & $260 \pm 26$ & $840 \pm 40$ & $390 \pm 100$ & $0.78 \pm 0.03$ & $0.11 \pm 0.08$ \\
$\left(\mathrm{Fe}_{(30 \mathrm{~nm})} / \mathrm{Cu}_{(120 \mathrm{~nm})}\right)$ & $30 \pm 3$ & $430 \pm 30$ & $280 \pm 35$ & $0.37 \pm 0.02$ & $0.20 \pm 0.02$ \\
$\left(\mathrm{Fe}_{(100 \mathrm{~nm})} / \mathrm{Cu}_{(120 \mathrm{~nm})}\right)$ & $100 \pm 8$ & $620 \pm 45$ & $260 \pm 50$ & $0.56 \pm 0.04$ & $0.17 \pm 0.03$ \\
$\left(\mathrm{Fe}_{(300 \mathrm{~nm})} / \mathrm{Cu}_{(120 \mathrm{~nm})}\right)$ & $300 \pm 60$ & $890 \pm 40$ & $363 \pm 70$ & $0.72 \pm 0.02$ & $0.09 \pm 0.03$ \\
\hline
\end{tabular}



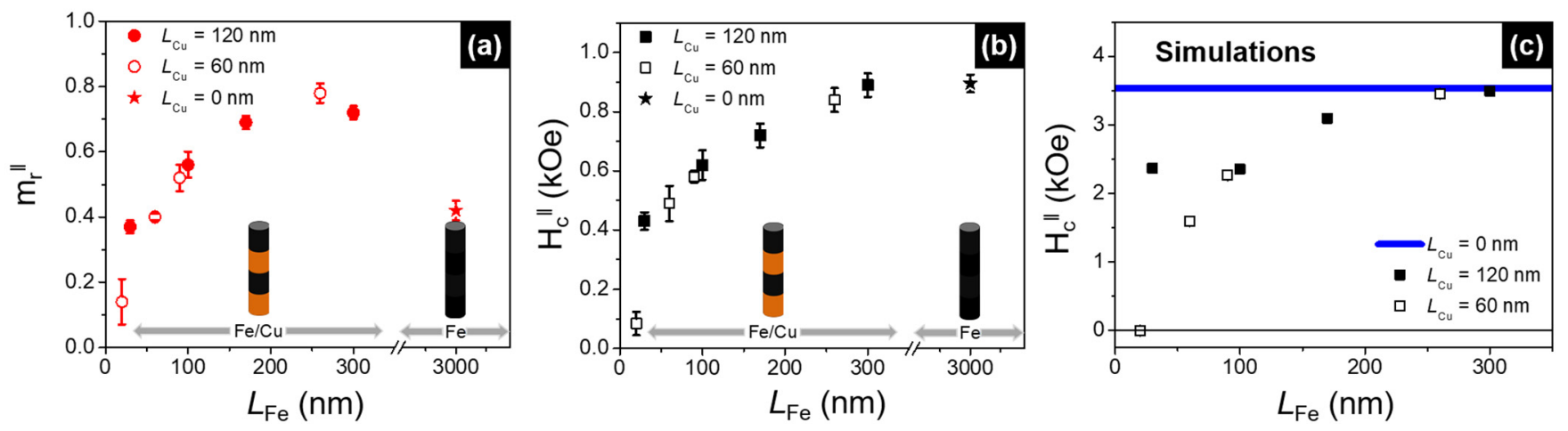

Figure 6. (a) Reduced remanence and (b) coercivity values as a function of the Fe length, measured when applying a magnetic field parallel to the wires' long axis. (c) Coercive field as a function of the Fe segment length in Fe/Cu NWs with

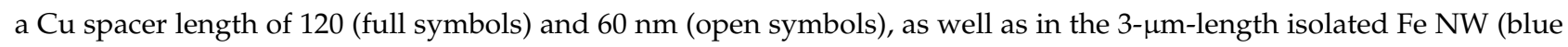
continuous line), extracted from the simulated hysteresis loops when the external field was applied parallel to the NW's longitudinal axis.

Regarding the evolution of the simulated coercive fields as a function of the Fe segment length for $\mathrm{Cu}$ spacer lengths of 60 and $120 \mathrm{~nm}$ (Figure 6c), these values progressively increased with the Fe length, approaching the value corresponding to the long Fe NW. Again, and despite having simulated only one wire, a good qualitative correlation with the experimental data was achieved, demonstrating that larger magnetostatic interactions are acting on the NWs when the Fe segments are longer.

\section{Conclusions}

In this work, bi-segmented multilayered Fe/Cu NWs have been successfully fabricated by pulsed electrodeposition in AAO templates, presenting a diameter of $45 \mathrm{~nm}$ and variable aspect ratios. Their morphological characterization revealed uniform and distinguishable layers, while the structural one showed a polycrystalline body-centered cubic (bcc) structure for either the Fe or $\mathrm{Cu}$ NWs. The magnetic measurements and micromagnetic simulations have demonstrated that the behavior of the Fe/Cu NWs can be easily tuned by increasing the number of layers and/or the ferromagnetic layer lengths. In this context, it was shown that the easy magnetization axis is mainly ruled by the shape anisotropy contribution. It was observed that the coercivity field values vary with the number of segments. Therefore, the required $H_{C}$ can be selectively obtained by controlling the number of bilayers, making it possible to achieve values ranging between the coercivity of a segmented NW with only one segment and the value observed for an Fe NW with a length of several micrometers.

Furthermore, the magnetic configuration at remanence can range from a single domain parallel to the longitudinal wire axis, for Fe lengths $\geq 60 \mathrm{~nm}$, to a vortex configuration, for NWs with Fe segments exhibiting lengths of $\approx 30 \mathrm{~nm}$. For layered NWs with Fe segments presenting $20 \mathrm{~nm}$ in length, the easy magnetization axis lies in the sample plane or perpendicular to the longitudinal wire axis, illustrating a synthetic antiferromagnet behavior with negligible coercivity and remanence values.

In summary, it was demonstrated that multilayered NWs can be fabricated with a well-controlled magnetic behavior. We believe that this possibility of achieving fine tuning and control of the magnetic response of multilayered NWs may be of great interest in technological applications that require high sensitivity and selectivity, e.g., for the development of magnetic sensor devices.

Author Contributions: Conceptualization, C.T.S., M.P.P. and D.N.; methodology, S.C., S.M. and R.M.; magnetic simulations, D.N.; validation, M.P.P., C.N. and J.P.A.; formal analysis, C.T.S. and D.N.; investigation, S.C. and S.M.; resources, J.P.A., M.P.P. and C.T.S.; writing-original draft preparation, S.C., S.M. and D.N.; writing-review and editing, C.T.S. and M.P.P.; supervision, C.T.S., C.N. and J.P.A.; project administration, C.T.S.; funding acquisition, J.P.A. and C.T.S. All authors have read and agreed to the published version of the manuscript. 
Funding: This work was financially supported by the FCT and COMPETE 2020 (FEDER) under the projects POCI-01-0141-FEDER-032527, PTDC/FIS-MAC/31302/2017, PTDC/CTM-CTM/28676/2017, and PTDC/FIS-OTI/32257/2017, UIDB/04968/2020 and UIDP/04968/2020 IFIMUP- Institute of Physics for Advanced Materials, Nanotechnology and Photonics-University of Porto, the Spanish Ministerio de Ciencia e Innovación under the project PID2020-117024GB-C42, and the European Union's Horizon 2020 research and innovation program under the Marie Sklodowska-Curie Grant Agreement No. 734801. R.M. and C.T.S. would like to acknowledge the Portuguese Fundação para a Ciência e Tecnologia (FCT), respectively, for grants SFRH/BD/110698/2015 and IF/01159/2015. D.N. wants to acknowledge the Spanish Ministerio de Ciencia, Innovación y Universidades for funding through the "Ramón y Cajal" program RYC-2017-22820. SM thanks CNPq (Brazil) for financial support through the grant No. 234513/2014-4.

Institutional Review Board Statement: Not applicable.

Informed Consent Statement: Not applicable.

Conflicts of Interest: The authors declare no conflict of interest.

\section{References}

1. Peng, X. Nanowires: Recent Advances; IntechOpen: London, UK, 2012.

2. Zhai, T.; Yao, J. One-Dimensional Nanostructures: Principles and Applications; Wiley: Hoboken, NJ, USA, 2012.

3. Fratila, R.M.; Rivera-Fernandez, S.; de la Fuente, J.M. Shape matters: Synthesis and biomedical applications of high aspect ratio magnetic nanomaterials. Nanoscale 2015, 7, 8233. [CrossRef] [PubMed]

4. Sarkar, J.; Khan, G.G.; Basumallick, A. Nanowires: Properties, applications and synthesis via porous anodic aluminium oxide template. Bull. Mater. Sci. 2007, 30, 271-290. [CrossRef]

5. Piraux, L. Magnetic Nanowires. Appl. Sci. 2020, 10, 1832. [CrossRef]

6. Peixoto, L.; Magalhães, R.; Navas, D.; Moraes, S.; Redondo, C.; Morales, R.; Araújo, J.P.; Sousa, C.T. Magnetic nanostructures for emerging biomedical applications. Appl. Phys. Rev. 2020, 7, 011310. [CrossRef]

7. Streubel, R.; Fischer, P.; Kronast, F.; Kravchuk, V.P.; Sheka, D.D.; Gaididei, Y.; Schmidt, O.G.; Makarov, D. Magnetism in curved geometries. J. Phys. D Appl. Phys. 2016, 49, 363001. [CrossRef]

8. Charilaou, M.; Braun, H.-B.; Löffler, J.F. Monopole-Induced Emergent Electric Fields in Ferromagnetic Nanowires. Phys. Rev. Lett. 2018, 121, 097202. [CrossRef]

9. Fernandez-Roldan, J.A.; del Real, R.P.; Bran, C.; Vazquez, M.; Chubykalo-Fesenko, O. Magnetization pinning in modulated nanowires: From topological protection to the "corkscrew" mechanism. Nanoscale 2018, 10, 5923-5927. [CrossRef] [PubMed]

10. Hertel, R. Curvature-Induced Magnetochirality. In SPIN; World Scientific: Singapore, 2013; Volume 3, p. 1340009.

11. Hertel, R. Ultrafast domain wall dynamics in magnetic nanotubes and nanowires. J. Phys. Condens. Matter 2016, $28,483002$. [CrossRef]

12. Xia, Y.; Yang, P.; Sun, Y.; Wu, Y.; Gates, B.; Yin, Y.; Kim, F.; Yan, H. One-Dimensional Nanostructures: Synthesis, Characterization, and Applications. Adv. Mater. 2003, 15, 353-387. [CrossRef]

13. Stepniowski, W.J.; Salerno, M. Fabrication of nanowires and nanotubes by anodic alumina template-assisted electrodeposition. In Manufacturing Nanostructures; One Central Press: Cheshire, UK, 2014; Volume 1, pp. 321-357.

14. Ertan, A.; Tewari, S.N.; Talu, O. Electrodeposition of nickel nanowires and nanotubes using various templates. J. Exp. Nanosci. 2008, 3, 287-295. [CrossRef]

15. Vázquez, M.; Hernández-Vélez, M.; Pirota, K.; Asenjo, A.; Navas, D.; Velázquez, J.; Vargas, P.; Ramos, C. Arrays of Ni nanowires in alumina membranes: Magnetic properties and spatial ordering. Eur. Phys. J. B 2004, 40, 489-497. [CrossRef]

16. Lee, W.; Park, S.-J. Porous Anodic Aluminum Oxide: Anodization and Templated Synthesis of Functional Nanostructures. Chem. Rev. 2014, 114, 7487-7556. [CrossRef]

17. Susano, M.; Proenca, M.P.; Moraes, S.; Sousa, C.T.; Araujo, J.P. Tuning the magnetic properties of multisegmented Ni/Cu electrodeposited nanowires with controllable Ni lengths. Nanotechnology 2016, 27, 335301. [CrossRef]

18. Böhnert, T.; Niemann, A.C.; Michel, A.-K.; Bäßler, S.; Gooth, J.; Tóth, B.G.; Neuróhr, K.; Péter, L.; Bakonyi, I.; Vega, V.; et al. Magnetothermopower and magnetoresistance of single Co-Ni/Cu multilayered nanowires. Phys. Rev. B 2014, 90, 165416. [CrossRef]

19. Moraes, S.; Navas, D.; Beron, F.; Proenca, M.P.; Pirota, K.R.; Sousa, C.T.; Araújo, J.P. The Role of Cu Length on the Magnetic Behaviour of Fe/Cu Multi-Segmented Nanowires. Nanomaterials 2018, 8, 490. [CrossRef] [PubMed]

20. ATehrani, S.; Kashi, M.A.; Ramazani, A.; Montazer, A.H. Axially adjustable magnetic properties in arrays of multilayered Ni/Cu nanowires with variable segment sizes. Superlattices Microstruct. 2016, 95, 38-47.

21. Maleak, N.; Potpattanapol, P.; Bao, N.N.; Ding, J.; Wongkokuo, W.; Tang, I.M.; Thongmee, S. Fabrication and magnetic properties of electrodeposited Ni/Cu nanowires using the double bath method. J. Magn. Magn. Mater. 2014, 354, 262-266. [CrossRef]

22. Béron, F.; Carignan, L.-P.; Ménard, D.; Yelon, A. Magnetic Behavior of Ni/Cu Multilayer Nanowire Arrays Studied by First-Order Reversal Curve Diagrams. IEEE Trans. Magn. 2008, 44, 2745. [CrossRef] 
23. Chen, M.; Searson, P.C.; Chien, C.L. Micromagnetic behavior of electrodeposited Ni/Cu multilayer nanowires. J. Appl. Phys. 2003, 93, 8253. [CrossRef]

24. Chen, M.; Chien, C.-L.; Searson, P.C. Potential Modulated Multilayer Deposition of Multisegment Cu/Ni Nanowires with Tunable Magnetic Properties. Chem. Mater. 2006, 18, 1595-1601. [CrossRef]

25. Clime, L.; Zhao, S.Y.; Chen, P.; Normandin, F.; Roberge, H.; Veres, T. The interaction field in arrays of ferromagnetic barcode nanowires. Nanotechnology 2007, 18, 435709. [CrossRef]

26. Ramazani, A.; Ghaffari, M.; Kashi, M.A.; Kheiry, F.; Eghbal, F. A new approach to fabricating magnetic multilayer nanowires by modifying the ac pulse electrodeposition in a single bath. J. Phys. D Appl. Phys. 2014, 47, 355003. [CrossRef]

27. Ohgai, T.; Hashiguchi, K.; Morimura, T.; Takao, K.; Kagawa, A. Fabrication of Co/Cu Multilayered Nanowires Using a Pulsed Current Deposition Technique. Mater. Sci. Forum 2010, 654-656, 1728-1731. [CrossRef]

28. Wong, J.; Greene, P.; Dumas, R.K.; Liu, K. Probing magnetic configurations in Co/Cu multilayered nanowires. Appl. Phys. Lett. 2009, 94, 032504. [CrossRef]

29. Park, B.C.; Kim, B.G.; Seo, H.W.; Kim, Y.K. Magnetic Anisotropy Evolution in CoFe/Au Barcode Nanowire Arrays. IEEE Trans. Magn. 2014, 50, 2500204. [CrossRef]

30. Ramazani, A.; Kashi, M.A.; Eghbal, F.; Jafari-Khamse, E. The effect of deposition parameters on the magnetic behavior of $\mathrm{CoFe} / \mathrm{Cu}$ multilayer nanowires. Eur. Phys. J. Plus 2015, 130, 1-8. [CrossRef]

31. Tang, X.-T.; Wang, G.-C.; Shima, M. Magnetic layer thickness dependence of magnetization reversal in electrodeposited CoNi/Cu multilayer nanowires. J. Magn. Magn. Mater. 2007, 309, 188-196. [CrossRef]

32. Akhtari-Zavareh, A.; Carignan, L.P.; Yelon, A.; Ménard, D.; Kasama, T.; Herring, R.; Dunin-Borkowski, R.E.; McCartney, M.R.; Kavanagh, K.L. Off-axis electron holography of ferromagnetic multilayer nanowires. J. Appl. Phys. 2014, 116, 023902. [CrossRef]

33. Tan, L.; Stadler, B.J.H. Fabrication and magnetic behavior of Co/Cu multilayered nanowires. J. Mater. Res. 2006, 21, 2870-2875 [CrossRef]

34. Xu, S.H.; Fei, G.T.; Zhu, X.G.; Zhang, L.D. Orientation-dependent growth rate of crystalline plane study in electrodeposited $\mathrm{Ni} / \mathrm{Cu}$ superlattice nanowires. Cryst. Eng. Comm. 2013, 15, 4070-4076. [CrossRef]

35. Sahin, T.; Kockar, H.; Alper, M. Properties of electrodeposited CoFe/Cu multilayers: The effect of Cu layer thickness. J. Magn. Magn. Mater. 2015, 373, 128-131. [CrossRef]

36. Núñez, A.; Pérez, L.; Abuín, M.; Araujo, J.P.; Proenca, M.P. Magnetic behaviour of multisegmented FeCoCu/Cu electrodeposited nanowires. J. Phys. D Appl. Phys. 2017, 50, 155003. [CrossRef]

37. Özkale, B.; Shamsudhin, N.; Chatzipirpiridis, G.; Hoop, M.; Gramm, F.; Chen, X.; Martí, X.; Sort, J.; Pellicer, E.; Pané, S. Multisegmented FeCo/Cu Nanowires: Electrosynthesis, Characterization, and Magnetic Control of Biomolecule Desorption. ACS Appl. Mater. Interfaces 2015, 7, 7389-7396. [CrossRef] [PubMed]

38. Bran, C.; Ivanov, Y.P.; Kosel, J.; Chubykalo-Fesenko, O.; Vazquez, M. Co/Au multisegmented nanowires: A 3D array of magnetostatically coupled nanopillars. Nanotechnology 2017, 28, 095709. [CrossRef]

39. Berganza, E.; Jaafar, M.; Bran, C.; Fernández-Roldán, J.A.; Chubykalo-Fesenko, O.; Vázquez, M.; Asenjo, A. Multisegmented Nanowires: A Step towards the Control of the Domain Wall Configuration; Scientific Reports: London, UK, 2017; Volume 7, p. 1 1576.

40. Wang, D.-S.; Mukhtar, A.; Wu, K.-M.; Gu, L.; Cao, X. Multi-Segmented Nanowires: A High Tech Bright Future. Materials 2019, 12, 3908. [CrossRef]

41. Almasi-Kashi, M.; Ramazani, A.; Kheyri, F.; Jafari-Khamse, E. The effect of magnetic layer thickness on magnetic properties of $\mathrm{Fe} / \mathrm{Cu}$ multilayer nanowires. Mater. Chem. Phys. 2014, 144, 230-234. [CrossRef]

42. Guo, Y.-G.; Wan, L.-J.; Zhu, C.-F.; Yang, D.-L.; Chen, D.-M.; Bai, C.-L. Ordered Ni-Cu Nanowire Array with Enhanced Coercivity. Chem. Mater. 2003, 15, 664-667. [CrossRef]

43. Gamburg, Y.D.; Zangari, G. Theory and Practice of Metal Electrodeposition; Springer: New York, NY, USA, 2011.

44. Gunawardena, G.; Hills, G.; Montenegro, I.; Scharifker, B. Electrochemical nucleation: Part I. General considerations. J. Electroanal. Chem. Interfacial Electrochem. 1982, 138, 225-239. [CrossRef]

45. Scharifker, B.; Hills, G. Theoretical and experimental studies of multiple nucleation. Electrochim. Acta 1983, 28, 879-889. [CrossRef]

46. Proenca, M.P.; Sousa, C.T.; Ventura, J.; Vazquez, M.; Araujo, J.P. Ni growth inside ordered arrays of alumina nanopores: Enhancing the deposition rate. Electrochim. Acta 2012, 72, 215-221. [CrossRef]

47. Jacobs, J.W.M. Note on a theory of three-dimensional electrochemical nucleation with diffusion-controlled growth. J. Electroanal. Chem. Interfacial Electrochem. 1988, 247, 135-144. [CrossRef]

48. Oskam, G.; Long, J.G.; Natarajan, A.; Searson, P.C. Electrochemical deposition of metals onto silicon. J. Phys. D Appl. Phys. 1998, 31, 1927-1949. [CrossRef]

49. Palmero, E.M.; Béron, F.; Bran, C.; del Real, R.P.; Vázquez, M. Magnetic interactions in compositionally modulated nanowire arrays. Nanotechnology 2016, 27, 435705. [CrossRef]

50. Vansteenkiste, A.; Leliaert, J.; Dvornik, M.; Helsen, M.; Garcia-Sanchez, F.; Waeyenberge, B.V. The design and verification of MuMax3. AIP Adv. 2014, 4, 107133. [CrossRef]

51. Xie, Y.-P.; Zhao, S.-J. The energetic and structural properties of bcc NiCu, FeCu alloys: A first-principles study. Comput. Mater. Sci. 2011, 50, 2586-2591. [CrossRef]

52. Palma, J.L.; Morales-Concha, C.; Leighton, B.; Altbir, D.; Escrig, J. Micromagnetic simulation of Fe asymmetric nanoring. J. Magn. Magn. Mater. 2012, 324, 637-641. [CrossRef] 
53. Roshchin, I.V.; Li, C.-P.; Suhl, H.; Batlle, X.; Roy, S.; Sinha, S.K.; Park, S.; Pynn, R.; Fitzsimmons, M.R.; Mejía-López, J.; et al. Measurement of the vortex core in sub-100 nm Fe dots using polarized neutron scattering. EPL (Europhys. Lett.) 2009, 86, 67008. [CrossRef]

54. Ivanov, Y.P.; Vázquez, M.; Chubykalo-Fesenko, O. Magnetic reversal modes in cylindrical nanowires. J. Phys. D Appl. Phys. 2013, 46, 485001. [CrossRef]

55. Haehnel, V.; Fähler, S.; Schaaf, P.; Miglierini, M.; Mickel, C.; Schultz, L.; Schlörb, H. Towards smooth and pure iron nanowires grown by electrodeposition in self-organized alumina membranes. Acta Mater. 2010, 58, 2330-2337. [CrossRef] 